\title{
Portal Vein Thrombosis and Cavernoma Formation in a Patient With Small Vessel Vasculitis
}

\author{
Muhammad Nadeem Iftikhar ${ }^{\mathrm{a}}$, Mureed Hussain ${ }^{\mathrm{b}}$, Vishal Kaushik ${ }^{\mathrm{c}}$, Sohail Iqbal ${ }^{\mathrm{d}, \mathrm{e}}$
}

\begin{abstract}
Caput medusa or palm tree sign refers to dilated tortuous paraumbilical veins acting as portosystemic shunt in patients with portal vein thrombosis (PVT). We present an asymptomatic male patient who was diagnosed to have chronic PVT with cavernoma formation. His initial presentation was acute exacerbation of chronic obstructive lung disease. He was known to have small vessel vasculitis resulting in ischemic bowel resection. Bowel ischemia and surgical intervention are predisposing factors of PVT which is underdiagnosed clinically and when diagnosed, is diagnosed with delay. Early diagnosis has a good prognosis and can potentially avert morbidity.
\end{abstract}

Keywords: Caput medusa; Palm tree sign; Portal vein thrombosis; Cavernoma formation; Small vessel vasculitis; Bowel ischemia

\section{Introduction}

Acute portal vein thrombosis (PVT) is often diagnosed incidentally at radiological studies or at presentation of its complications such as gastrointestinal bleeding, ascites or hypersplenism [1], because of non-specific presentation and low sensitivity of the grayscale transabdominal ultrasound in assessing the portal vein. PVT occurs due to portal pyemia secondary to intra-abdominal infections, abdominal surgery,

\footnotetext{
Manuscript accepted for publication August 13, 2015

aAcute Medicine \& Gastroenterology, Royal Oldham Hospital, The Pennine Acute Hospitals NHS Trust, Oldham, OL1 2JH, UK

${ }^{b}$ Acute Medicine, Royal Oldham Hospital, The Pennine Acute Hospitals NHS Trust, Oldham, OL1 2JH, UK

${ }^{\mathrm{c}}$ Gastroenterology, Royal Blackburn Hospital, East Lancashire Hospitals NHS Trust, Haslingden Road, Blackburn, BB2 3HH, UK

dRadiology Department, Royal Preston Hospital, Lancashire Teaching Hospitals NHS Foundation Trust, Preston Lancashire, PR2 9HT, UK formerly working at Royal Blackburn Hospital, East Lancashire Hospitals NHS Trust, Haslingden Road, Blackburn, BB2 3HH, UK

${ }^{\mathrm{e}}$ Corresponding Author: Sohail Iqbal, 4-Tagore Close Manchester, M13 0YS, UK. Email: drsohailiqbal@yahoo.com
}

doi: http://dx.doi.org/10.14740/jmc2272w myeloproliferative disorders, pancreatitis, liver cirrhosis, malignancy, trauma, inflammatory bowel disease, hypercoagulable states, and venous stasis secondary to cardiac abnormalities such as constrictive pericarditis and long-term use of oral contraceptives. A cavernoma is a constellation of peri-portal varices and is a sign of chronic PVT.

\section{Case Report}

A 52-year-old male smoker was admitted to hospital to receive treatment for an acute exacerbation of chronic obstructive pulmonary disease. On examination, it was noted that he had grossly dilated veins on the abdominal wall with centrifugal flow. There were no other clinical signs of chronic liver disease and no hepato-splenomegaly. The patient had a history of small bowel resection for an ischemic ileum, multiple lung infarcts, popliteal artery embolism and two deep vein thromboses. The thrombophilia screen was negative and a diagnosis of small vessel vasculitis was confirmed on the resected bowel histology. His autoimmune profile was normal. He was taking warfarin and steroids in addition to other medications.

The abdominal examination revealed caput medusa which is recanalization of the umbilical veins radiating away from the

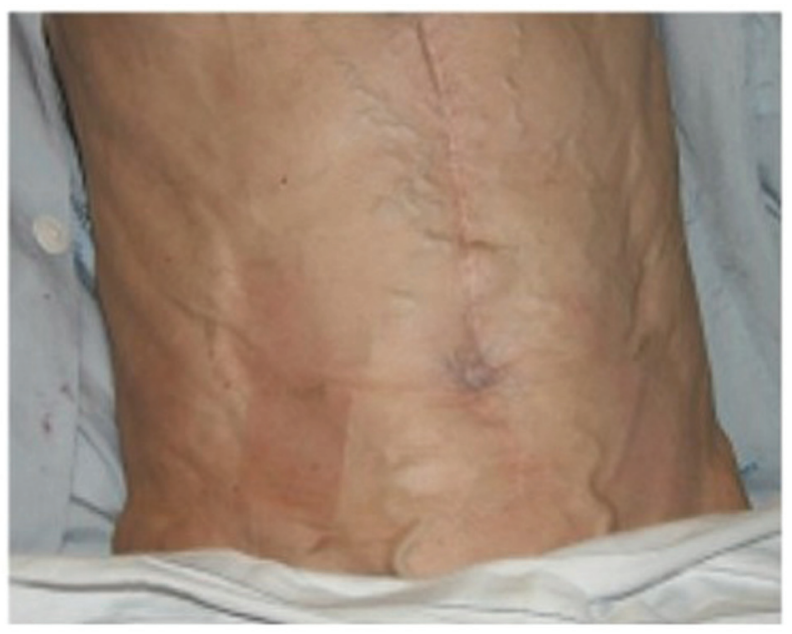

Figure 1. Dilated and tortuous veins in the anterior abdominal wall radiating away from the umbilicus known as caput medusa. 


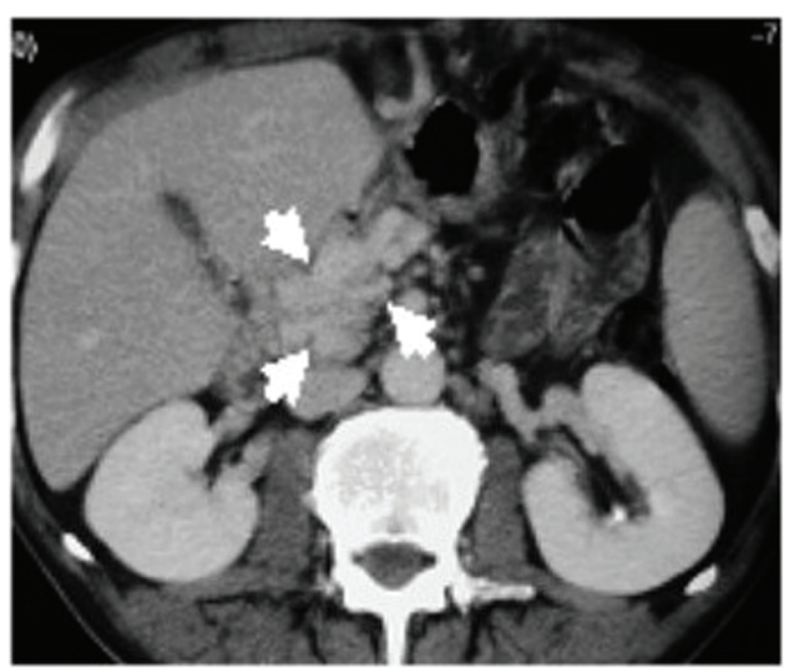

Figure 2. CECT axial section reveals cavernoma formation in the extrahepatic portal vein marked by arrow heads.

umbilicus (Fig. 1). It is seen in conditions that cause portal hypertension with intrahepatic or posthepatic venous occlusion. The color Doppler scan and contrast-enhanced computerized tomography (CECT) scan (Fig. 2) depicted cavernoma formation instead of normal portal vein. The patient probably developed PVT and posthepatic venous occlusive disease due to high thrombotic tendency from vasculitis and intra-abdominal infection related to his ischemic bowel and subsequent surgery. However, it went unrecognized until noted on the color Doppler ultrasound and CT scan.

\section{Discussion}

Cavernous formation of the portal vein is a reparative process to bypass the already thrombosed native portal vein. It can arise in the intrahepatic branches of the portal vein or in the main portal vein itself secondary to occluding or non-occluding thrombus and helps in the continued flow of blood even in the presence of a clot [2]. It is actually recanalization of thrombosed veins which has been formed within 6 - 20 days after the formation of acute PVT [3]. It is a phenomenon to help keep liver to retain its functioning in a normal way; however, if this cavernous formation is unable to handle mesenteric and splenic inflow, the formation of pre-sinusoidal portal hypertension or cirrhosis ensues. Obstruction of portal veins was first diagnosed in 1869 by Stewart and Balfour in a patient with variceal dilation, enlarged spleen and ascites [4].

Clinical presentation depends on the timing and progression of thrombosis and collateral formation. Acute PVT patients may present with nausea, fever, vomiting, hematochezia and abdominal pain due to ischemia and intestinal congestion. Chronic PVT patients are usually asymptomatic. This may lead to cirrhosis and ascites. Associated findings include splenomegaly, anemia, leucopoenia, jejunal varices, and gastrointestinal bleeding. Portal biliopathy or cholestatic syndrome may develop because of external compression of the bile duct by convoluted vessels or from stricture formation in biliary ducts. This is observed in $80 \%$ of chronic PVT.

Diagnostic pearls in grayscale ultrasound are filling defect in the portal vein which is hypoechoic in acute phase and echogenic in chronic phase. It may reveal abnormal growth of portal venous channels and can be supplemented with color Doppler which has $90 \%$ sensitivity of PVT diagnosis [4]. The diagnosis may also be confirmed by performing a CT scan and/or MRI with greater anatomical details. CECT shows non-enhanced intraluminal filling defect with rim enhancement. The MRI shows hyperintense signal on T2 sequence. Endoscopic retrograde cholangiopancreatography (ERCP) may reveal a false impression of cholangiocarcinoma, known as the pseudocholangiocarcinoma sign [5]. Pre-operative and during transjugular intrahepatic portosystemic shunt (TIPS), portal venography depicts filling defect. Traditionally chronic portal vein thrombosis (CPVT) was diagnosed by the clue of recanalization of umbilical veins in the anterior abdominal wall.

Therapeutic options include careful management of underlying causes and complications of the disease such as banding of esophageal varices and shunting procedures [6]. Acute presentations are treated with anti-coagulant therapy. Alternatively thrombolytic agents can be instilled into the portal vein directly. Surgical removal of the thrombus reduces the chance of chronic PVT disease. It is curable with $61 \%$ and $54 \%$ survival rates at 5 and 10 years respectively. The outcome is poorer for patients having cirrhosis and malignancy, and in patients with myelofibrotic transformation or acute leukemia and hepatic failure.

\section{Conclusion}

Prompt diagnosis of PVT and cavernous transformation of the portal vein in symptomatic patients with abdominal pain, gastrointestinal bleeding, portal pyaemia, and rapid onset of ascites in acute stages without hepato-splenomegaly or chronic liver disease can have excellent outcome. Imaging has wider impacts on therapy planning. Treatment options vary from anti-coagulant therapy, surgical re-construction or removal of thrombus and reduction of portal pressure by TIPS and active management of complications. It is critical to differentiate the malignant portal vein thrombosis from other causes due to poor prognosis of the curative resections.

\section{References}

1. Sheen CL, Lamparelli H, Milne A, Green I, Ramage JK. Clinical features, diagnosis and outcome of acute portal vein thrombosis. QJM. 2000;93(8):531-534.

2. Boyer TD. Management of portal vein thrombosis. Gastroenterol Hepatol (N Y). 2008;4(10):699-700.

3. Ma J, Yan Z, Luo J, Liu Q, Wang J, Qiu S. Rational classification of portal vein thrombosis and its clinical significance. PLoS One. 2014;9(11):e112501.

4. Ramos R, Park Y, Shazad G, C AG, Cohen R. Cavernous transformation of portal vein secondary to portal vein thrombosis: a case report. J Clin Med Res. 2012;4(1):81- 
84.

5. Rosu A, Searpe C, Sbarcea V, Stoica Z, Popescu M. A case of portal cavernoma--associated essential thrombocythemia. J Gastrointestin Liver Dis. 2007;16(1):97-100.
6. Webster GJM, Burroughs AK, Riordan SM. Review article: portal vein thrombosis - new insights into aetiology and management. Alimentary Pharmacology \& Therapeutics. 2005;21:1-9. 